\title{
Del derecho a la alimentación al derecho al cuidado nutricional
}

\author{
From the right to food to the right to nutritional care \\ Do direito à alimentação ao direito ao cuidado nutricional
}

\author{
Diana Cardenas ${ }^{1 *}$, Sonia Echeverri², Charles Bermúdez ${ }^{3}$
}

Recibido: 1 de septiembre de 2019. Aceptado para publicación: 26 de octubre 2019. https://doi.org/10.35454/rncm.v2supl1.029

\begin{abstract}
Resumen
La desnutrición es un problema de salud pública en el mundo. La desnutrición asociada a factores socioeconómicos es competencia del ámbito del derecho a la alimentación adecuada. El abordaje de este tipo de desnutrición se logra a través de las dos vertientes de este derecho: el derecho a estar protegido contra el hambre y el derecho a una alimentación adecuada que implica la necesidad de constituir un entorno económico, político y social que permita a las personas alcanzar la seguridad alimentaria por sus propios medios. Sin embargo, la desnutrición asociada a la enfermedad no puede definirse dentro del alcance del derecho a la alimentación. En este contexto, debe considerarse como un derecho humano al cuidado nutricional donde la persona en riesgo o en estado de desnutrición pueda recibir cuidado nutricional incluyendo la terapia nutricional de manera óptima y oportuna. Este nuevo derecho humano emergente debe ser estudiado y definido desde el enfoque de los derechos humanos para que sea reconocido ante las instituciones de derechos humanos internacionales y nacionales.
\end{abstract}

Palabras clave: desnutrición, salud públi$\mathrm{ca}$, derecho al cuidado nutricional.

\section{Summary}

Malnutrition is a public health problem in the world. Malnutrition associated with socioeconomic factors falls within the scope of the right to adequate food. The approach to this type of malnutrition is achieved through the two aspects of this right: the right to be protected against hunger and the right to adequate food, wich implies the need to constitute an economic, political and social environment that allows people to achieve food security by their own means. However, malnutrition associated with the disease cannot be defined within the scope of the right to food. In this context, a human right to nutritional care should be considered where the person at risk or in a state of malnutrition can receive nutritional care including nutritional therapy in an optimal and timely manner. This new emerging human right must be studied and defined from the human rights approach to be recognized before international and national human rights institutions.

Keywords: Malnutrition; Public health; Right to nutritional care.

\section{Resumo}

A desnutrição é um problema de saúde pública no mundo. A desnutrição associada a factores socioeconómicos encontra-se no domínio do direito à alimentação adequada. A abordagem para esse tipo de desnutrição é alcançada através dos dois aspectos desse direito: o direito a ser protegido contra a fome e o direito a alimentação adequada, o que implica a necessidade de constituir um ambiente económico, político e social que permita as pessoas alcançarem a segurança alimentar por seus próprios meios. No entanto, a desnutrição associada à doença não pode ser definida no âmbito do direito à alimentação. Nesse contexto, um direito humano aos cuidados nutricionais deve ser considerado onde a pessoa em risco ou em estado de desnutrição pode receber cuidados nutricionais, incluindo terapia nutricional, de maneira ótima e oportuna. Esse novo direito humano emergente, deve ser estudado e definido a partir da abordagem de direitos humanos para ser reconhecida perante instituições nacionais e internacionais de direitos humanos.

Palavras-chave: desnutrição, saúde pública, direito ao cuidado nutricional.
1 Instituto de Investigación en Nutrición, Genética y Metabolismo, Facultad de Medicina, Universidad El Bosque, Bogotá, D.C., Colombia.

2 Comité de Ética Hospitalaria y Comité de Humanismo y Bioética de la Fundación Santa Fe de Bogotá, Bogotá, D.C., Colombia.
3 Departamento de Cirugía. Clínica la Colina y Clínica del Country. Bogotá, Colombia. Presidente ACNC 2017-2021.

*Correspondencia: Diana Cardenas dianacardenasbraz@gmail.com 


\section{INTRODUCCIÓN}

La malnutrición es un problema de salud pública en el mundo debido al alto impacto sobre la morbilidad, la mortalidad y los costos para los sistemas de salud. Según la Organización Mundial de la Salud (OMS) la malnutrición puede ser de tres tipos: sobrepeso/obesidad, desnutrición y alteraciones de micronutrientes. En la Figura 1 se muestra la clasificación de los trastornos nutricionales ${ }^{(1)}$. La desnutrición en la Clasificación Internacional de Enfermedades 11 (ICE11) se encuentra dentro del código 5 "enfermedades endocrinas" y como parte de los "trastornos nutricionales". La des- nutrición se define allí como "un trastorno en el cual los requerimientos del organismo no se ven satisfechos debido a un consumo insuficiente de nutrientes o a deficiencia de su absorción o aprovechamiento. Puede deberse a una falta de acceso a alimentos o a una enfermedad. Por «desnutrición» por lo general, se entiende como un déficit del consumo energético, pero también puede hacer referencia a la carencia de nutrientes específicos. Puede ser aguda o crónica ${ }^{(2)}$.

En esta definición podemos identificar dos grandes tipos de desnutrición por déficit. La primera, la desnutrición asociada a factores socioeconómicos y al hambre donde el punto de partida es el acceso inadecuado a los

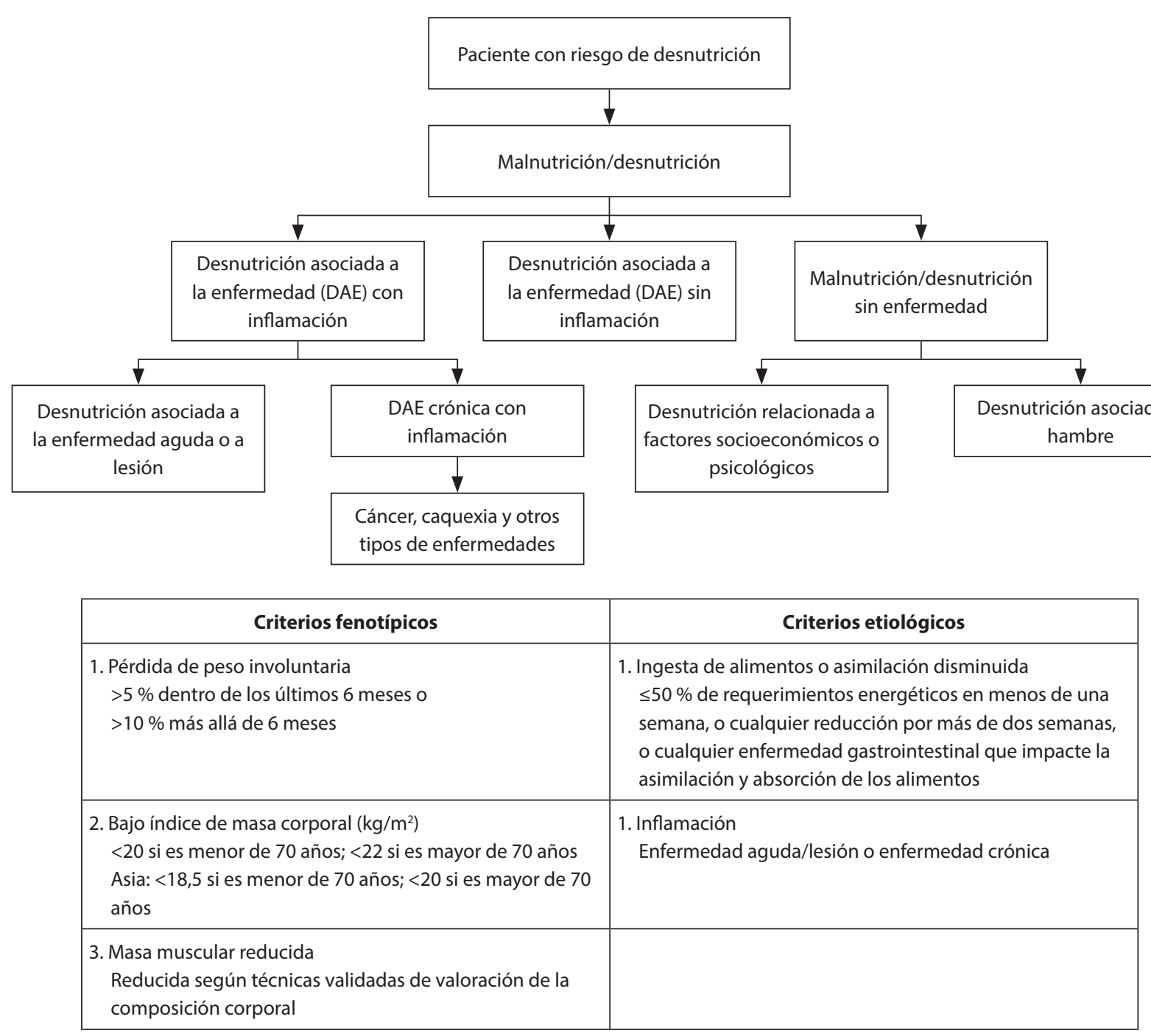

Figura 1. Clasificación de los trastornos nutricionales según la Sociedad Europea de Nutrición Clínica y Metabolismo (ESPEN), $2017^{(1)}$ y los criterios diagnósticos Global Leadership Initiative on Malnutrition (GLIM) de la desnutrición asociada a la enfermedad, 2018. Según ESPEN, en inglés undernutrition (desnutrición) es sinónimo de malnutrition (malnutrición). 
alimentos (Figura 2). Las cifras según la Organización de las Naciones Unidas para la Alimentación y la Agricultura (FAO) muestran que, en el año 2017, 821 millones de personas sufrían de hambre, es decir, 1 de cada 9 personas en el mundo. El enfoque para abordar este problema se basa en las políticas públicas de nutrición y en la erradicación de la pobreza y el hambre ${ }^{(3)}$. El enfoque de derechos humanos desde hace varias décadas, a través del derecho a la alimentación adecuada, ha permitido que los Estados se responsabilicen y actúen para proteger a las poblaciones contra el hambre y la malnutrición.

La segunda, la desnutrición asociada a la enfermedad donde el punto de partida son los grados variables de inflamación aguda o crónica asociados a la enfermedad o al trauma, y las consecuentes adaptaciones metabólicas, la ingesta reducida de alimentos o la disminución de su asimilación ${ }^{(1)}$ (Figura 3). Esto conlleva a alteración de la composición corporal, con pérdida de masa magra, de tejido adiposo y a una función biológica disminuida ${ }^{(1)}$. Para abordar la desnutrición asociada a la enfermedad, el cuidado nutricional ha demostrado ser un proceso eficaz y costo-efectivo ${ }^{(4,5)}$. Sin embargo, a pesar de esto y de que hoy es técnicamente posible de administrar terapia nutricional a cualquier persona enferma, la prevalencia de la desnutrición asociada a la enfermedad al ingreso hospitalario sigue siendo alta, entre $40 \%$ y $60 \%$ según los distintos estudios ${ }^{(6)}$. Las políticas públicas y las legislaciones para abordar esta problemática son escasas y el enfoque desde los derechos humanos nunca se ha estudiado.

Frente a este problema nos cuestionamos: ipor qué el derecho a la alimentación no es respetado en el ámbito clínico? Por sorprendente que pueda parecer, en el hospital, lugar privilegiado del cuidado a la persona donde se busca la salud y el máximo nivel de bienestar de las personas, este derecho es irrespetado con demasiada frecuencia. Nuestra hipótesis es que la desnutrición asociada a la enfermedad no entra en el ámbito del concepto ni del contenido normativo del derecho a la alimentación. En este artículo mostraremos que en el contexto clínico y en cualquier nivel de atención en salud, el derecho a la alimentación no puede ser garantizado debido a que su contenido normativo no aplica.

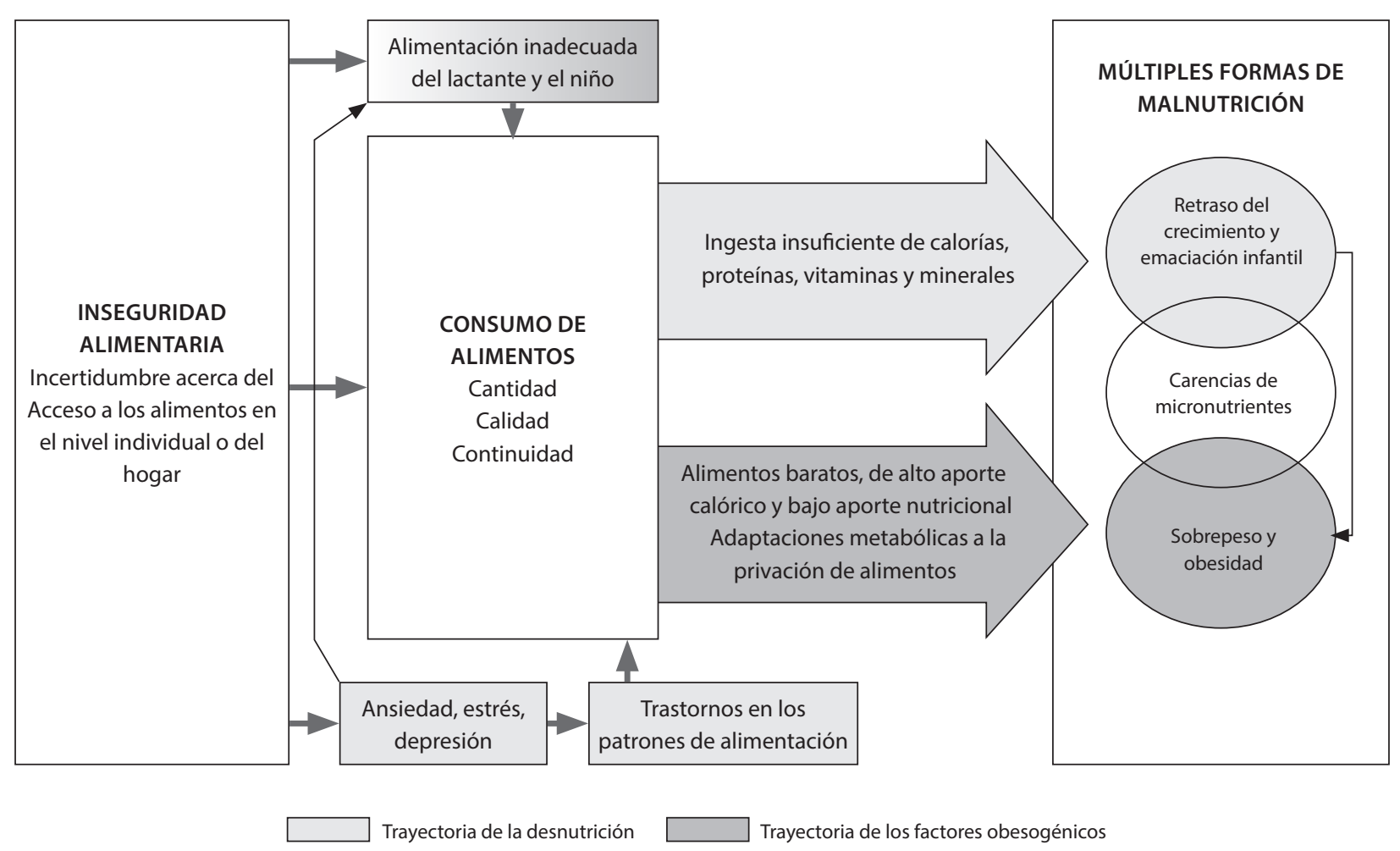

Figura 2. Trayectoria del acceso inadecuado a los alimentos a las múltiples formas de malnutrición, según la FAO, 2018 $8^{(8)}$. 


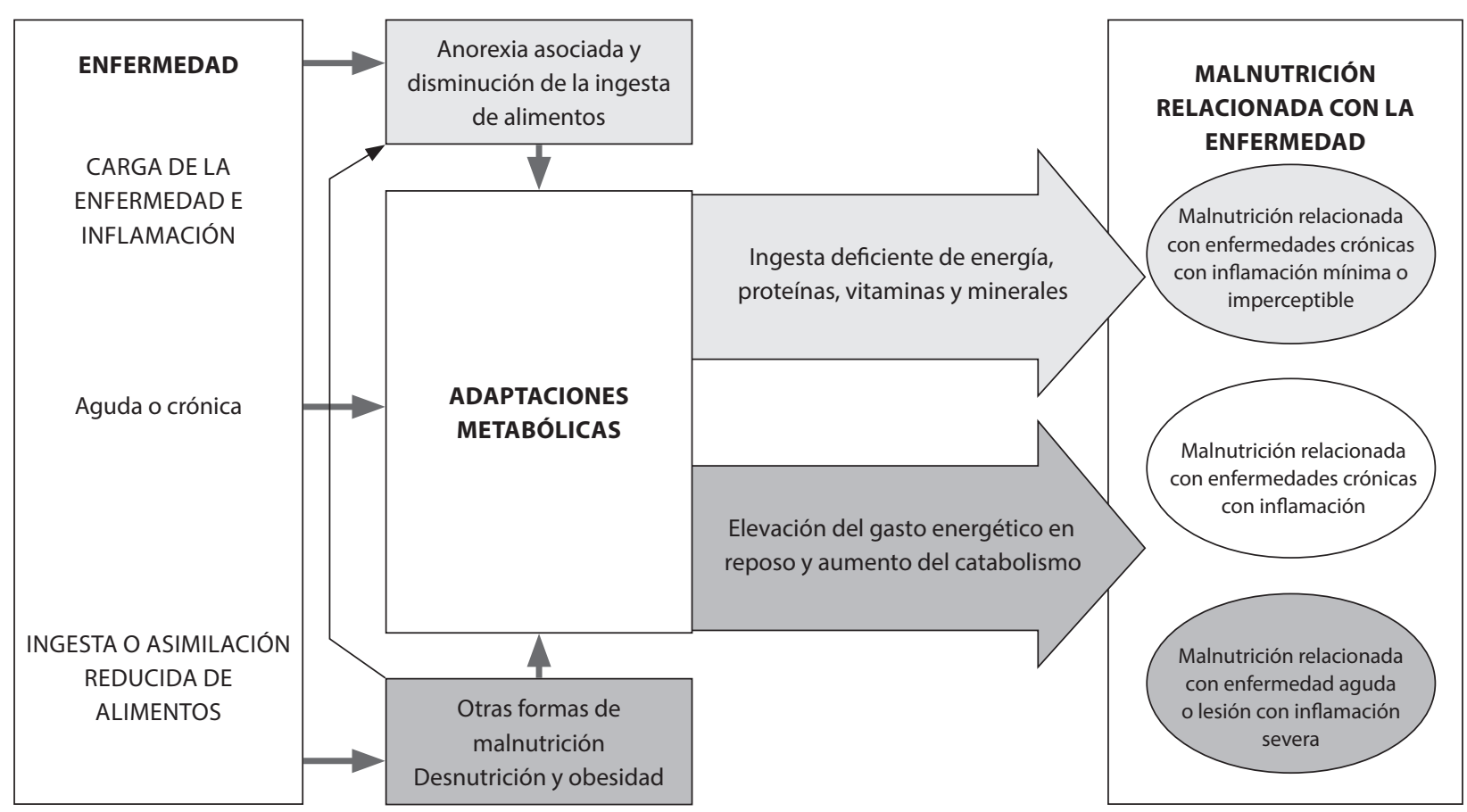

Figura 3. Trayectoria de la enfermedad a las diversas formas de desnutrición. Figura basada en la definición y clasificación de la desnutrición ${ }^{(1)}$.

Por lo tanto, es necesario reconocer un nuevo derecho humano emergente: el derecho al cuidado nutricional.

\section{EL DERECHO A LA ALIMENTACIÓN}

El derecho a una alimentación adecuada como un derecho humano fundamental fue reconocido por primera vez, en el marco del derecho a un nivel de vida adecuado, en la Declaración Universal de Derechos Humanos de 1948 (Artículo 25):

"Toda persona tiene derecho a un nivel de vida adecuado para la salud, el bienestar y el bienestar de su familia, incluidos los alimentos...." ${ }^{\prime 7}$. Este derecho se convirtió en legalmente vinculante (que obliga a las partes) cuando el Pacto Internacional de los Derechos Económicos, Sociales y Culturales (PIDESC) entró en vigor en 1976. Desde entonces, otros acuerdos internacionales han reiterado el derecho a la alimentación, incluida la Convención sobre la eliminación de todas las formas de discriminación contra la mujer (1979), la Convención Internacional sobre los Derechos del Niño (1989), la Convención sobre el Estatuto de los Refugiados
(1951), la Convención sobre el Derecho de las Personas con Discapacidad (2006) y diversos instrumentos regionales de derechos humanos. Hasta la fecha, 160 Estados han ratificado el PIDESC y, por lo tanto, están legalmente obligados a aplicar sus disposiciones. En el artículo 11 de este Pacto se establece que los Estados parte «reconocen el derecho de toda persona a un nivel de vida adecuado para él y su familia, incluida la alimentación adecuada»y afirma la existencia del derecho de toda persona a estar protegida contra el "hambre y la malnutrición” ${ }^{\prime(8)}$.

Vivir una vida sin hambre es considerado como el mínimo que debe garantizar cada Estado independiente del nivel de desarrollo ${ }^{(14)}$. Sin embargo, el derecho a la alimentación no se limita a este aspecto. El Comité de Derechos Económicos, Sociales y Culturales ha definido el derecho a la alimentación en su comentario general No. 12 como:

"El derecho a una alimentación adecuada se realiza cuando cada hombre, mujer y niño, solo o en comunidad, tiene física y económicamente en 
todo momento acceso a alimentos suficientes o a los medios para obtenerlos"(9).

Además, el Comité subraya que el derecho a una alimentación adecuada "no debe interpretarse restrictiva o estrictamente como el derecho a una ración mínima de calorías, proteínas u otros nutrientes específicos". También se deben tener en cuenta otros elementos como las prácticas alimentarias, la educación en temas de higiene, la formación en nutrición, la provisión de cuidados de salud y la lactancia. Esto implica que cada persona debe tener acceso a los alimentos no solo para no sufrir de hambre sino para tener un buen estado de salud y bienestar. Por lo tanto, este derecho engloba dos normas distintas: la primera es el derecho a "una alimentación adecuada", la segunda es que "toda persona esté protegida contra el hambre y la malnutrición” ${ }^{(8)}$.

Este enfoque del derecho a la alimentación, que ha ido evolucionando desde la década de los años 90, pretende ir más allá del enfoque de seguridad alimentaria que hasta entonces se ha defendido. Ya no se trata solo de la defensa y la promoción de la producción agrícola y de garantizar la disponibilidad de los alimentos; ahora se trata de un derecho a la alimentación que permite proteger a las personas del hambre y la malnutrición para lograr un buen estado de salud. Es decir, se busca proteger el derecho a satisfacer las propias necesidades alimentarias, ya sea produciendo o comprando los productos. Se considera un cambio radical de perspectiva: el ciudadano beneficiario del derecho a la alimentación ya no es un receptor indefenso, pasivo, un objeto de caridad, y el cual debe "ser alimentado", sino una persona que tiene derecho a beneficiarse de un entorno que le permite alimentarse y, en su defecto, recibir asistencia no solo con dignidad sino con calidad ${ }^{(10)}$.

En conclusión, la desnutrición asociada a factores socioeconómicos entra en el ámbito del derecho a la alimentación adecuada. El abordaje de este tipo de desnutrición se logra a través de las dos vertientes de este derecho. Por un lado, el derecho a estar protegido contra el hambre considerado como una norma absoluta, $y$ como el nivel mínimo que debe garantizarse a todas las personas independientemente del nivel de desarrollo alcanzado por el Estado. Por otro lado, el derecho a una alimentación adecuada el cual abarca mucho más, ya que conlleva la necesidad de constituir un entorno económico, político y social que permita a las personas alcanzar la seguridad alimentaria por sus propios medios.

¿La desnutrición asociada a la enfermedad tiene cabida en el derecho humano a la alimentación? ¿Cómo debe entenderse el derecho a la alimentación en el contexto clínico? En otras palabras, la pregunta fundamental es ise debe garantizar el derecho de las personas a «alimentarse $» 0$ a ser «alimentadas»?

\section{EL DERECHO AL CUIDADO NUTRICIONAL: UN DERECHO HUMANO EMERGENTE}

Recordemos que en el ámbito de la salud pública y en el contexto político se considera que el beneficiario o titular del derecho a la alimentación es una persona con un papel activo a la cual el Estado debe proporcionar un entorno propicio para permitirle «alimentarse a sí mismo» y, en su defecto, recibir asistencia con dignidad. En el contexto clínico, ¿se debe garantizar el derecho de las personas a «alimentarse a si mismas» o a ser «alimentadas»?

En el contexto clínico, la persona enferma tiene mayor probabilidad de presentar alteración del estado nutrición por el simple hecho de estar enfermo. Por lo tanto, toda persona enferma en contacto con una institución de salud debería poder recibir cuidado nutricional. Es decir, se debería realizar tamizaje nutricional para identificar el riesgo nutricional, un diagnóstico preciso de la desnutrición que conlleva a un plan nutricional que incluye la alimentación y la terapia nutricional, su vigilancia y monitoreo (Figura 4). Para que el paciente se beneficie del cuidado nutricional es indispensable un profesional experto en nutrición clínica para asegurar un completo y adecuado proceso de cuidado nutricional. La persona enferma no puede decidir por si sola el tipo de nutrición o alimentación que necesita. Es el profesional quien emite una recomendación y una indicación terapéutica. Por supuesto que es el paciente, y su autonomía, quien dispone de libertad para decidir si acepta o no lo propuesto por el profesional de la salud.

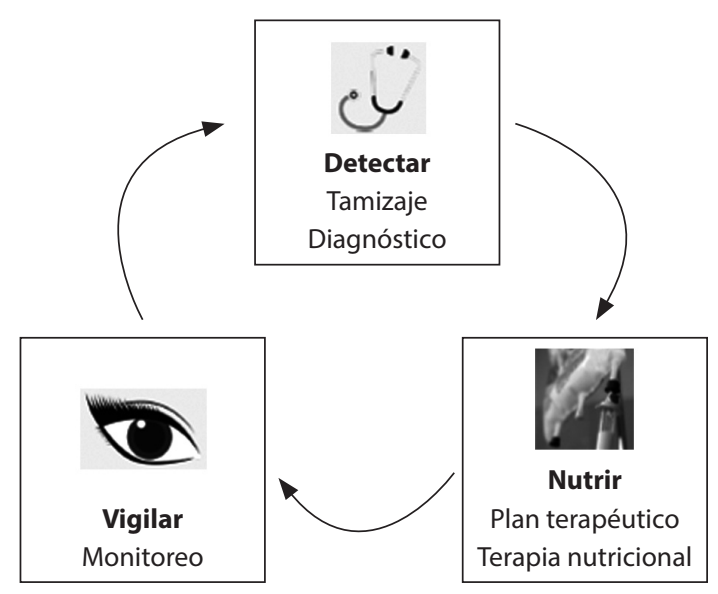

Figura 4. Las etapas del proceso de cuidado nutricional. 
Por lo tanto, la persona enferma tiene el derecho a recibir una terapia nutricional es decir "a ser alimentado" y esto debe estar garantizado por el Estado y los cuidadores. El contenido normativo del derecho a la alimentación tal y como es concebido en los instrumentos internacionales de derechos humanos, no puede ser aplicado de la misma manera en el contexto clínico. Así las cosas, es posible reconocer que los pacientes tienen el derecho a recibir un cuidado nutricional óptimo y oportuno, y en consecuencia, es posible reconocer un derecho humano emergente: el derecho al cuidado nutricional (Figura 5).

Reconocer la existencia de un derecho al cuidado nutricional, tendría implicaciones para los beneficiarios del derecho (los pacientes desnutridos o en riesgo de desnutrición), y para los titulares de deberes como el Estado y los legisladores. Para los pacientes, este derecho implicaría que puede reclamar al estado un cuidado nutricional completo que permita prevenir o al menos limitar las alteraciones nutricionales y modular las adaptaciones metabólicas para tener un impacto positivo en la evolución de la enfermedad. Para los Estados, este derecho implicaría garantizar como mínimo el acceso, la aceptabilidad, la disponibilidad y la calidad de alimentos y la terapia nutricional en el hospital (Figura 5).

Para los profesionales de la salud, el derecho al cuidado nutricional debe guiar sus acciones. Se trata de defender el derecho de cada persona enferma a recibir todas las etapas del cuidado nutricional que conlleven al paciente a «ser alimentado» en condiciones de dignidad. Más específicamente, el derecho a beneficiarse del cuidado nutricional y a recibir los requerimientos nutricionales a través de una terapia nutricional oportuna, óptima y de calidad, en un contexto que respalde la dimensión emocional, simbólica y social de la alimentación. Se debe hacer énfasis en que alimentar a la persona enferma no debe ser considerado un acto de caridad, como lo fue cuando se alimentaba a los enfermos en el hospital durante la Edad Media. Por el contrario, debe considerarse como un cuidado y un tratamiento médico que se integra al tratamiento general de los pacientes. En consecuencia, los médicos, los nutricionistas, los enfermeros y otros profesionales de la salud deben proteger, respetar y garantizar el derecho al cuidado nutricional y esto a través de la realización de todas las etapas del cuidado nutricional incluyendo una terapia nutricional oportuna y óptima.

\section{CONCLUSIÓN}

La desnutrición asociada a la enfermedad no puede definirse dentro del alcance del derecho a la alimentación. El derecho a la alimentación tal y como es considerada en los instrumentos internacionales de
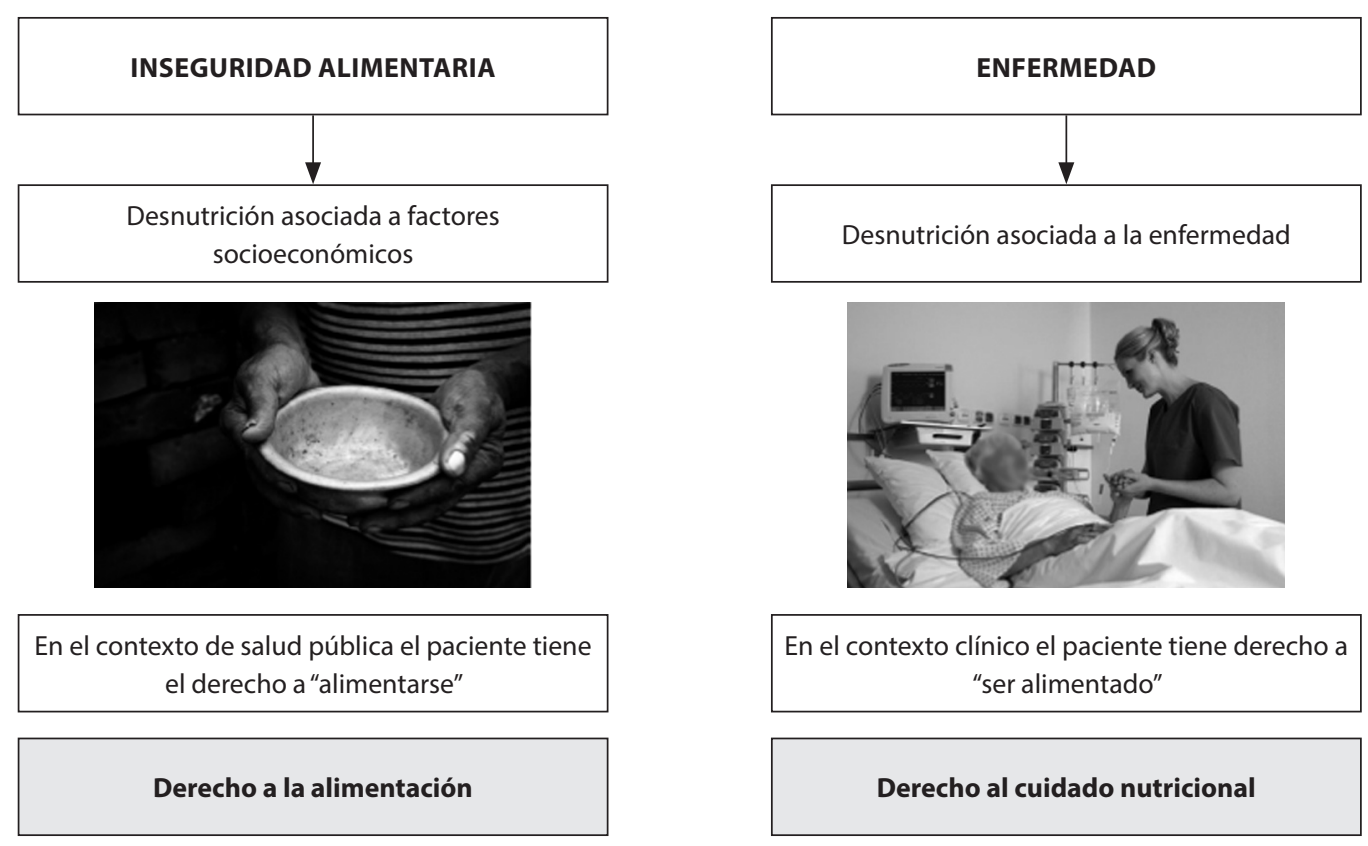

Figura 5. El derecho a la alimentación versus el derecho al cuidado nutricional. 
derechos humanos, como el derecho a "alimentarse" no puede ser garantizado de la misma manera en el ámbito clínico. En este contexto, debe considerarse un derecho humano al cuidado nutricional donde la persona en riesgo o en estado de desnutrición reciba cuidado nutricional incluyendo la terapia nutricional de manera óptima y oportuna. Este nuevo derecho humano emergente debe ser estudiado y definido desde el enfoque de los derechos humanos para que sea reconocido ante las instituciones de derechos humanos nacionales e internacionales.

\section{Financiación}

El presente artículo no tuvo financiación.

\section{Conflicto de intereses}

Los autores declaran no tener conflicto de intereses.

\section{Declaración de autoría}

Los autores declaran que revisaron de artículo y validaron su versión final.

\section{Referencias bibliográficas}

1. Cederholm T, Barazzoni R, Austin P, Ballmer P, Biolo G, Bischoff SC, et al. ESPEN guidelines on definitions and terminology of clinical nutrition. Clin Nutr. 2017;36(1):49-64.

2. World Health Organization. [Internet]. International Classification of Diseases 11th Revision. The global standard for diagnostic health information ICD 11. (Consultado el 5 de septiembre de 2019). Disponible en: https://icd.who.int/en.

3. Food and Agriculture Organization of the United Nations. The state of food security and nutrition in the world. [Internet]. Roma 2018. (Consultado el 5 de septiembre de 2019). Disponible en: http://www.fao.org/3/I9553ES/i9553es.pdf.

4. Rosen BS, Maddox P, Ray N. A position paper on how cost and quality reforms are changing healthcare in America: focus on nutrition. JPEN J Parenter Enteral Nutr. 2013;37:796-801.

5. Kris-Etherton PM, Akabas SR, Bales CW, Bistrian B, Braun L, Edwards MS, Laur C, Lenders CM, Levy MD, Palmer CA, et al. The need to advance nutrition education in the training of health care professionals and recommended research to evaluate implementation and effectiveness. Am J Clin Nutr. 2014;99(Suppl.):1153S-66S.

6. Correia MITD, Perman MI, Waitzberg DL. Hospital malnutrition in Latin America: A systematic review. Clin Nutr. 2016;36:958-67.

7. Declaración Universal de Derechos Humanos de 1948. [Internet]. (Consultado el 1 de agosto 2019). Disponible en: http://www. un.org.

8. Food and Agriculture Organization of the United Nations. [Internet]. Le droit à l'alimentation. Le temps d'agir Avancées et enseignements tirés lors de la mise en application. Roma 2012. (Consultado el 1 de agosto 2019). Disponible: http:// www.fao.org/docrep/016/i2250f/ i2250f.pdf.

9. Le droit à l'alimentation. Organisation des nations unies pour l'alimentation et l'agriculture, Rome 2006 [Internet]. (Consultado el 1 de agosto 2019). Disponible: http://www. fao.org/3/a-ah189f.pdf.

10. Diouf J. Droit á l'alimentation, notions generales. FAO, 2007. [Internet]. (Consultado el 1 de agosto 2019). Disponible: http://www1.montpellier.inra.fr/aide-alimentaire/images/. 